\title{
A RARE VULVAL MASS
}

Reethu Varadarajan¹, Nirmala Shivalingaiah ${ }^{2}$.

1. Assistant Professor, Department of OBG, KIMS Bangalore.

2. Professor, Department of OBG, KIMS Bangalore

\section{CORRESPONDING AUTHOR:}

Dr. Reethu Varadarajan

No. 55, 6 th main road

Chamrajpet, Bangalore.

E-mail: reethu.nandeesh23@gmail.com

Aggressive angiomyxoma (AAM) is an uncommon distinctive mesenchymal tumor with a predilection for the pelvic and perineal regions in females. Since its initial description in 1983 by Steeper and Rosai, approximately 65 cases have been reported in English literature ${ }^{(1,2)}$. The term 'Aggressive' has been added to this mesenchymal tumour to denote its propensity for local aggression, reticent recurrences and lack of metastatic potential(3). We report one such case at our institute.

CASE REPORT: A 65 year old postmenopausal patient presented with mass per vagina since 6 months. Initially the mass was small in size gradually progressed to the present size. She had no other relevant complaints. She is known case of diabetes on treatment. On examination her vitals were stable. Local examination revealed a huge pedunculated swelling of about $25 \times 15 \mathrm{~cm}$ arising from left labia majora. Skin over the swelling was stretched with dilated veins, there was no ulceration/pulsations. It was non tender with no local rise in temperature. It had a soft cystic feel and was freely mobile. On per speculum, cervix was flushed with vagina. Per vaginum , uterus was retroverted atrophic, mobile, non tender. Her blood investigations were within normal limits. Scan showed uterus atrophic with normal adnexa. Patient underwent wide local excision of the mass, which was sent for histopathology. The gross appearance is shown in figure one. Postoperatively, there were no complications. Histopathology study of the excised mass showed features of aggressive angiomyxoma of vulva.

DISCUSSION: Carcinoma of the vulva is an uncommon malignancy accounting for $2 \%$ of all female genital malignancies. It is predominantly a disease of older women between 16 and 70 years(mean age: 34) and about one-fourth of these tumours are pedunculated (4). The predominant histological type is squamous cell carcinoma which accounts for about $90 \%$ of the tumors. Primary sarcoma constitutes $1 \%$ to $3 \%$ of all vulvar malignancies. The most common histological type of primary vulvar sarcoma is leiomyosarcoma.

The line of differentiation of this tumour is not firmly established, but a fibroblastic / myofibroblastic origin has been proposed (5) .AAM is a mesenchymal tumor that affects women in $>90 \%$ of cases.(4,6) It may involve the pelvis, retroperitoneum, vagina, vulva perineum, or buttocks, and usually presents as a polyploid or cystic mass.(7)The size is also variable, but most are larger than 10 
cm. ${ }^{(4)}$ It is a slow growing tumor, but problematic due to frequent local recurrences (30-72\%), sometimes even decades later.

The differential diagnoses ranges from benign tumors such as myxolipoma, myxoid neurofibroma and myxoid leiomyoma to myxofibrosarcoma, myxoid variant of liposarcoma, leiomyosarcoma, malignant fibrous histiocytoma and botryoid rhabdomyosarcoma.

The tumors are lobulated, soft to rubbery, and on cut section have a gelatinous appearance. Some have finger-like projections that extend into neighbouring tissues. Although invasions are rare, AAM can invade the bladder, bowel and pelvic bone.(6) Histologically, the tumor consists of hypocellular, somewhat satellite to fusiform cells with thin cytoplasmic processes. The cells are scattered in a loose myxoid matrix composed of delicate wary collagen fibrils, which gives the tumor a pale-pink color by eosin staining. There is also a prominent vascular component, ranging from tiny vessels to larger vessels with a distinct smooth muscle cell, but with no evidence of anastomosis or arborization.

On immunohistochemistry, these tumors express estrogen and progesterone receptors, suggesting that they may be hormone dependent, hence rapid growth has been observed during pregnancy.(8,9). The tumour usually recurs in 30 to $40 \%$ of patients undergoing local excision, within a period of 10 months to 7 years (2). MRI is the best modality to detect recurrences.

The definitive treatment of AAM, is only a wide local excision. With repeated recurrences, a potential medical treatment with GnRH analogues may obviate the need for repeated surgeries for a recurrent aggressive angiomyxoma of the vulva (10).

CONCLUSION: Although a rare diagnosis, AAM can present with unusual features. Detailed radiological examination is helpful in detecting the problem, but histology is the gold standard for diagnosis. Wide excisions are curative and prognosis of such tumors is good. Long-term follow-up is necessary and MRI is the preferred method for detecting recurrences $(11,12)$. The patient we report has thus far done well with no clinical or radiographic recurrence.

\section{REFERENCES:}

1. Steeper TA, Rosai J: Aggressive angiomyxoma of the pelvis and perineum: report of nine cases of a distinctive type of gynaecologic soft tissue neoplasm. Am J Clin Pathol 1983; 7: 453.

2. Amezcua CA, Begley SJ, Mata $\mathrm{N}$ et al : Aggressive angiomyxoma of the female genital tract : a clinico-pathologic and immuno-histochemical study of 12 cases. Int J Gynecol Cancer 15(1) ; 140-5, 2005

3. Dash S, Awasthi RT, Bandana K: Pedunculated angiomyxoma of the vulva - A rare clinical entity. Kathmandu University Medical Journal 2005, Vol. 3, No. 4, Issue 12, 423-424

4. Fetsch JF, Laskin WB, Lefkowitz $M$ et al : Aggressive angiomyxoma : a clinicopathological study of 29 female patients. Cancer 78(1) : 79-90, 1996

5. Granter SR, Nucci MR, Fletcher CD: Aggressive angiomyxoma: reappraisal of its relationship to angiomyofibroblastoma in a series of 16 cases. Histopathology $30(1) ; 3-10$, 1997 
6. Smith HO, Worrell RV, Smith AY, Dorin MH, Rosenberg RD, Bartow SA: Aggressive angiomyxoma of the female pelvis and perineum: review of the literature. Gynecol Oncol 1991 42:79-85.

7. Awad Z, O’Dowd G, Sproule J, Ryan T, O’Reilly T. Aggressive angiomyxoma. Ir Med J 1999; 92: 343

8. Havel G, Burian P, Kortz M, Mark J: Aggressive angiomyxoma of the vulva: an unusual, deceptive and recurrence-prone tumour with evidence of estrogen receptor expression. Case report. APIMS 102(3);236-40, 1994

9. Htwe M, Deppisch LM, Saint-Julien JS: Hormone dependent, aggressive angiomyxoma of the vulva. Obstet Gynecol 86(4 Pt 2); 697-9,1995

10. Fine BA, Munoz AK, Litz CE et al: Primary medical management of recurrent angiomyxoma of the vulva with a gonadotropin-releasing hormone agonist. Gynecol Oncol 81(1); 120-2, 2001

11. Dragoumis K, Drevelengas A, Chatzigeorgiou K, Assimakopoulos E, Venizelos I, Togaridou E, et al: Aggressive angiomyxoma of the vulva extending into the pelvis: Report of two cases. J Obstet Gynaecol Res 2005; 31: 310-3.

12. Sharma JB, Wadhwa L, Malhotra, Arora R, Singh S. recurrent aggressive angiomyxoma of vagina: A case report. Indian J Pathol Microbiol 2004; 47: 425-7.

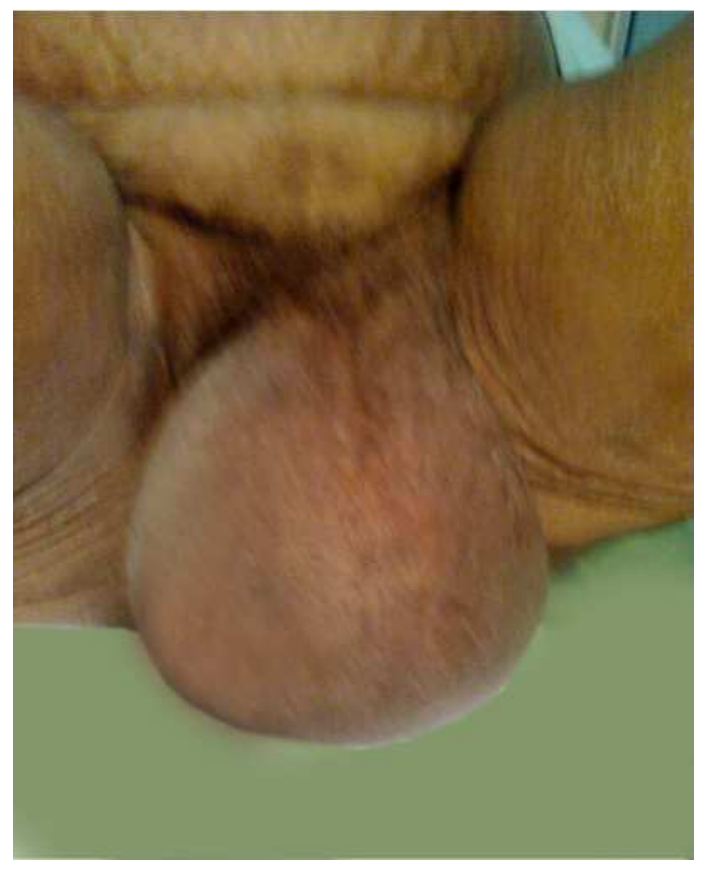

Figure-1 